\title{
Hepatocyte nuclear factor-6: associations between genetic variability and Type II diabetes and between genetic variability and estimates of insulin secretion
}

\author{
A.M. Møller ${ }^{1}$, J. Ek $k^{1}$, S. M. Durviaux ${ }^{3}$, S. A. Urhammer ${ }^{1}$, J. O. Clausen ${ }^{1}$, H. Eiberg ${ }^{2}$, T. Hansen ${ }^{1}$, G. G. Rousseau ${ }^{3}$, \\ F.P. Lemaigre ${ }^{3}$, O. Pedersen ${ }^{1}$ \\ ${ }^{1}$ Steno Diabetes Center and Hagedorn Research Institute, Gentofte, Copenhagen, Denmark \\ ${ }^{2}$ University Institute of Medical Biochemistry and Genetics, Department of Medical Genetics, University of Copenhagen, \\ Copenhagen, Denmark \\ ${ }^{3}$ Hormone and Metabolic Research Unit, Catholic University of Louvain and Christian de Duve Institute of Cellular Pathology, \\ Brussels, Belgium
}

\section{Abstract}

Aims/hypothesis. The transcription factor hepatocyte nuclear factor (HNF)-6 is an upstream regulator of several genes involved in the pathogenesis of maturity-onset diabetes of the young. We therefore tested the hypothesis that variability in the HNF-6 gene is associated with subsets of Type II (non-insulin-dependent) diabetes mellitus and estimates of insulin secretion in glucose tolerant subjects.

Methods. We cloned the coding region as well as the intron-exon boundaries of the $H N F-6$ gene. We then examined them on genomic DNA in six MODY probands without mutations in the MODY1, MODY3 and MODY4 genes and in 54 patients with late-onset Type II diabetes by combined single strand conformational polymorphism-heteroduplex analysis followed by direct sequencing of identified variants. An identified missense variant was examined in association studies and genotype-phenotype studies.
Results. We identified two silent and one missense (Pro75Ala) variant. In an association study the allelic frequency of the Pro 75 Ala polymorphism was $3.2 \%$ (95\% confidence interval, $1.9-4.5)$ in 330 patients with Type II diabetes mellitus compared with $4.2 \%$ (2.4-6.0) in 238 age-matched glucose tolerant control subjects. Moreover, in studies of 238 middle-aged glucose tolerant subjects, of 226 glucose tolerant offspring of Type II diabetic patients and of 367 young healthy subjects, the carriers of the polymorphism did not differ from non-carriers in glucose induced serum insulin or C-peptide responses.

Conclusion/interpretation. Mutations in the coding region of the HNF-6 gene are not associated with Type II diabetes or with changes in insulin responses to glucose among the Caucasians examined. [Diabetologia (1999) 42: 1011-1016]

Keywords Hepatocyte nuclear factor-6, Type II diabetes mellitus, MODY, insulin secretion, mutations.
Hepatocyte nuclear factors (HNFs) were identified through their interaction with DNA sequences. These sequences are essential for the liver-specific transcription of genes. Many of HNFs are also expressed in other tissues of endodermal origin [1]. Mu-

Received: 30 November 1998 and in final revised form: 17 February 1999

Corresponding author: A.M. Møller, Ms Sci, Steno Diabetes Center, Niels Steensens Vej 2, DK-2820 Gentofte, Copenhagen, Denmark

Abbreviations: HNF, hepatocyte nuclear factor; MODY, maturity-onset diabetes of the young; SSCP, single strand conformational polymorphism. tations in the $H N F-4 \alpha$ gene are associated with a subtype of maturity-onset diabetes of the young (MODY) denoted MODY1 [2], those in the HNF$1 \alpha$ gene are associated with MODY3 [3] and those in the $H N F-1 \beta$ gene with MODY5 [4]. In addition to an early onset ( $<25$ years of age) and an autosomal dominant inheritance, MODY1 and MODY3 are characterized by a primary defect in pancreatic betacell function. These findings raise the possibility that mutations in related transcription factors as well as in transcription factors implicated in the same regulatory cascade could cause other forms of Type II diabetes. Hepatocyte nuclear factor- 6 which defines the new ONECUT class of homeodomain proteins [5], contains a bipartite DNA-binding region consisting 
of a cut domain and of a variant homeodomain [6]. This factor stimulates the transcription of the $H N F$ $4 \alpha$ gene [7]. It also stimulates the transcription of the $H N F-3 \beta$ gene $[7,8]$, whose product in turn stimulates the transcription of the insulin promoter factor-1 gene [9], in which mutations are linked to MODY4 [10]. During pancreas development HNF-6 is expressed in the protodifferentiated epithelium, which contains the precursors of the beta-cells and which coexpresses $\mathrm{HNF}-3 \beta$ and insulin promoter factor- 1 $[7,8]$. In liver, HNF-6 controls the synthesis of enzymes that regulate glucose metabolism [6]. The human HNF-6 gene has been assigned to chromosome 15 [11] but only a cDNA corresponding to part of the coding region of the HNF-6 mRNA has been reported in humans [8]. The purpose of this study was to clone the complete coding region of the human $H N F-6$ gene as well as exon-intron junctions and to determine whether mutations in this gene are associated with Type II diabetes or with changes in the function of beta-cells in glucose tolerant Danish Caucasians.

\section{Subjects and methods}

Subjects. In the primary screening for mutations, combined single strand conformational polymorphism(SSCP)-heteroduplex analysis followed by nucleotide sequencing of migration variants was carried out on genomic DNA from six unrelated Caucasian MODY patients without mutations (examined by direct sequencing of the respective genes) in the MODY1, MODY3 and MODY4 genes and 54 patients with Type II (non-insulindependent) diabetes mellitus of late onset. The Type II diabetic patients were those with the lowest concentrations of fasting serum $C$ peptide selected from a group of 330 Danish Type II diabetic patients. The subsequent association study was done on this group of 330 unrelated Caucasian patients who were recruited from the outpatient clinic at Steno Diabetes Center, Copenhagen. It was also done on 238 age-matched and glucose tolerant Danish Caucasian control subjects traced randomly in the Danish Central Population Register. They lived in the same area of Copenhagen as the Type II diabetic patients but were not related to them or to each other. Type II diabetes was diagnosed by the 1985 World Health Organisation criteria. The Type II diabetic patients (141 women, 189 men) had a clinical onset of diabetes of $54.9 \pm 10.7$ years (mean \pm SD), an average duration of diabetes of $9.6 \pm 5.9$ years, fasting plasma glucose of $9.9 \pm 3.5 \mathrm{mmol} / \mathrm{l}$, fasting serum C peptide of $678 \pm 301 \mathrm{pmol} / \mathrm{l}$ and $\mathrm{HbA}_{1 \mathrm{c}}$ of $8.4 \pm 1.6 \%$ (interval for normal subjects: $4.1-6.4 \%)$. They all tested negative for GAD65 antibodies. The effect of genetic variants on beta-cell function was measured in 238 normal glucose tolerant middle-aged subjects, 226 glucose tolerant offspring of Type II diabetic probands from 62 Danish Caucasian families and in a population-based sample of 367 Danish Caucasians aged 18-32 years (Table 1). The study protocols were approved by the ethics committee of Copenhagen and were in accordance with the principles of the Declaration of Helsinki II. Prior to participation in the study informed consent was obtained for all subjects.

Biochemical variables. The plasma concentration of glucose was determined by an automated glucose oxidase method
(Granutest: Merck, Darmstadt, Germany). The concentration of insulin (excluding des(31, 32)- and intact proinsulin) in serum was measured by ELISA and the concentration of serum $\mathrm{C}$ peptide was determined by radioimmunoassay (RIA) using Steno Diabetes Centre routine methods [12-15].

Measurements of beta-cell function and insulin sensitivity index. The 238 control subjects and the 226 offspring of Type II diabetic patients in the case-control studies underwent a standard 75-g OGTT. Each of the 367 young subjects and the 226 glucose tolerant offspring in the phenotype studies underwent an intravenous glucose tolerance test (IVGTT, $0.3 \mathrm{~g}$ glucose per $\mathrm{kg}$ body weight) in combination with intravenous injection of $3 \mathrm{mg}$ tolbutamide per $\mathrm{kg}$ body weight at time $20 \mathrm{~min}$ ) after a 12 -h overnight fasting period as described [16]. Immediate serum insulin and $\mathrm{C}$ peptide responses (0-8 $\mathrm{min})$ induced by glucose were calculated by means of the trapezoidal rule as the incremental values (area under the curve when expressed above basal values). The insulin sensitivity index was calculated in accordance to Bergmann's minimal model as described [16].

Radiation hybrids. The human HNF-6 gene was mapped by PCR using genomic DNA from a panel of 93 radiation hybrids (human $\times$ hamster; Genebridge 4 Radiation Hybrid panel, Research Genetics). Genomic DNA (50 ng) was added in each PCR reaction $(50 \mu \mathrm{l})$ containing $1.25 \mathrm{U}$ of Taq polymerase (Qiagen, Leusden, The Netherlands) with the buffer conditions indicated by the supplier. We did 35 cycles $\left(95^{\circ} \mathrm{C}, 30 \mathrm{~s}\right.$; $60^{\circ} \mathrm{C}, 30 \mathrm{~s} ; 72^{\circ} \mathrm{C}, 60 \mathrm{~s}$ ) and the products were made visible on ethidium bromide-stained agarose gels. Two primer sets were used. The primers in set 1 were $5^{\prime}$-AGGAAAGAACAAGAACATGGGAAGG-3' (HNF-6.3) and 5'-GCTAGACGAAAATCACACTCC-3' (HNF-6.6). The primers in set 2 were $5^{\prime}$-AAACCAAAGACTTAGCTCACCTGCA$3^{\prime}$ (HNF-6.5) and primer HNF-6.6.

Cloning of the human HNF-6 gene. To clone the exon/intron junctions, human genomic DNA (600 ng) was cut with BanII or Sau3AI and the fragments were circularized through ligation. The 5 '-exon/intron junction was amplified with primers VLIIB (5'-CAGCCACTTCCACATCCTCCG-3') and $260 \mathrm{~S}$ (5 'AGCCGGAGTTCCAGCGCATGTC-3') using the circularized BanII-cut genomic DNA as a template. The intron $/ 3^{\prime}$ exon junction was amplified with primers HNF-6-RKE (5'CTTCCCGTGTTCTTGCTCTTTCC-3') and HNF-6.5 using the circularized Sau3AI-cut genomic DNA. The PCR amplifications were done with Expand High Fidelity polymerase (Boehringer, Brussels, Belgium) according to the supplier's instructions. The PCR products were subcloned in pBluescript KS (Stratagene, Amsterdam, The Netherlands) and sequenced. The $5^{\prime}$-end of the coding sequence was cloned after screening of $3.75 \times 10^{5}$ plaque forming units of a human genomic library in Lambda Dash II (Stratagene). The library screening procedure followed the supplier's instructions and was done using a 320-bp human cDNA fragment that spans residues 232 to 339 as a probe. Five clones were obtained and fragments were subcloned in pBluescript KS (Stratagene) to enable sequencing. These sequences have been deposited in the GenBank. (accession no. Y17739 and Y17740).

Primary analysis of the HNF-6 gene for mutations. The coding region as well as the intron-exon boundaries were examined in 8 segments of 200-350 bp by combined SSCP and hetereoduplex scanning with two different experimental settings as described previously [18]. The segments were amplified using specific primers as follows: segment 1: HNF6-1f, $5^{\prime}$-GCCA- 
Table 1. Clinical and biochemical characteristics of 238 middle-aged glucose tolerant Danish Caucasians, 367 young healthy Caucasian subjects and 226 glucose tolerant Caucasian off-

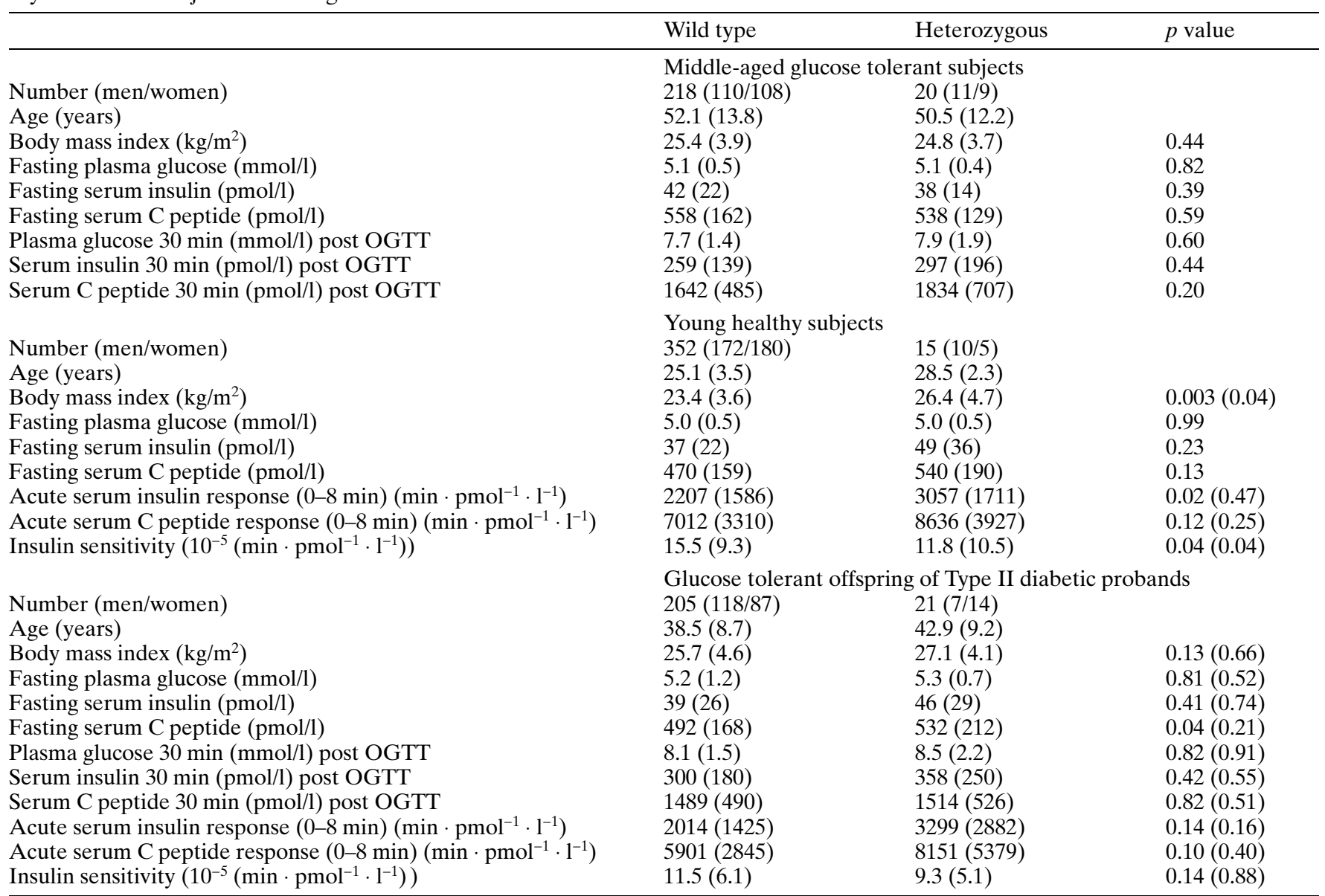

Data are means (SD). $P$ values compare wild-type subjects to heterozygous carriers of the Pro75Ala variant in the HNF-6 gene with regard to specific phenotypes. For young healthy subjects: $p$ values in parentheses were obtained after correction in a multiple regression analysis for differences in age,

CATCGATGTTGTGT-3', HNF6-1 r, 5'-TCCAGCAGGGACGCCATG-3'; segment 2: HNF6-2f, 5'-CGGCAGCCACCTGCCCCCCG-3', HNF6-2r, 5'-TGGTGGGGGAACTTGTCCGAG-3'; segment 3: HNF6-3f, 5'-CCTACACCACCTTGACCCCTC-3', HNF6-3 r, 5'-TGCTGCCCAGACCGGAGCT-3'; segment 4: HNF6-4f, 5'-TACCCCCTACCACAAGGAC-3', HNF6-4 r, 5 '-AGGAAGGCCGTTGATGGGCAC-3 '; segment 5: HNF6-5f, 5 '-TCGGCCGCCACGGGGAGC-3', HNF6-5r, 5'-GTAGCGCTTGAGCTCGGTGGT-3'; segment 6: HNF6-6f, 5'-GATGGAAGAGATCAATACC-3', HNF6-6r, 5'-GGCTTTCGTGTACCTTATC-3'; segment 7: HNF6-7f, 5'-TCTCCCCTAGACCATCTGTT-3', HNF6-7r, 5'-CGTTCATGAAGAAGTTGCTG-3'; segment 8: HNF6-8f, 5'-GCAAATCACCATTTCCCACC-3', HNF6-8 r, 5'-TCTAAGTATAAACCTGCTATC$3^{\prime}$. Polymerase chain reaction was carried out using AmpliTaq polymerase (Perkin Elmer, Foster City, Calif., USA) according to suppliers instructions. The conditions were: denaturation at $94^{\circ} \mathrm{C}$ for 3 min followed by 35 cycles of denaturation for $30 \mathrm{~s}$, annealing at $58^{\circ} \mathrm{C}$ (segment 6$), 60^{\circ} \mathrm{C}$ (segments $\left.1,7,8\right), 64^{\circ} \mathrm{C}$ (segment 5), $65^{\circ} \mathrm{C}$ (segments 2,4 ), or $66^{\circ} \mathrm{C}$ (segment 3 ) for 30 $\mathrm{s}$ and extension at $72^{\circ} \mathrm{C}$ for $30 \mathrm{~s}$, with a final extension at spring of Type II diabetic probands when classified according to genotype of the Pro75Ala polymorphism of the HNF-6 gene
BMI and sex between the two groups. The $p$ value in parentheses for BMI was corrected for differences in age and sex between the two groups. For glucose tolerant offspring of Type II diabetic probands: $p$ values in parentheses were obtained in a variance component model (see text)

$72^{\circ} \mathrm{C}$ for $9 \mathrm{~min}$. Variants identified by the SSCP and heteroduplex scanning were examined by nucleotide sequencing. PCRproducts used for sequencing were amplified applying the above mentioned primers except for the addition of a $21 \mathrm{M} 13$ tail at the 5 'end of the forward primer and a M13 reverse tail at the 5 'end of the reverse primer. The PCR products were sequenced on both strands using an ABI Prism Dye Primer Cycle Sequencing Kit with Amplitaq DNA Polymerase FS and ABI Prism 373 (Perkin Elmer).

Screening for amino acid polymorphisms in the HNF-6 gene. Polymerase chain reaction amplification of the DNA segment containing the Pro75Ala polymorphism was done using the forward primer 5 '-CTGCCCCCCGCGCACCCGGGCCCC3 ' and the reverse primer 5 '-GTAGGTGGTGGGCATGCTCATAC-3'. Restriction fragment length polymorphism analysis was applied after digestion with Bsp120I, which cuts both the wildtype allele and a control site introduced by the forward primer (mismatched bases indicated by bold letters). Polymerase chain reaction fragments were separated on $4 \%$ agarose gels and made visible by staining with ethidium bromide. 
Statistics. Chi-square analysis and Fishers exact test when appropriate were applied to test for significance of differences in allele frequencies. Differences in continuous variables between groups of subjects were tested with Student's $t$ test when the distributions of the variables or the logarithmic values of the variables were normal and when the variances of the variables were equal in the groups compared. Otherwise the Mann-Whitney test was used. To control for possible confounders on BMI, immediate serum insulin response, immediate serum C-peptide response and insulin sensitivity index, multiple linear regression analyses were carried out. Sex, age and BMI (only in the regression analysis of immediate serum insulin response, immediate serum $\mathrm{C}$-peptide response and insulin sensitivity index) were entered as explanatory variables and immediate serum insulin response, immediate serum Cpeptide response and insulin sensitivity index were entered as response variables. Also, for the glucose tolerant offspring of Type II diabetic patients, differences between groups were analysed applying a variance component model (random effects model) [19] where an extra source of variation is allowed to account for the fact that members of the same family might be correlated. The variant of interest and sex were included as fixed variables, age and BMI were included as covariates and family was included as a random effect. A $p$ value less than 0.05 (two-tailed) was considered significant.

\section{Results}

Using radiation hybrid analysis, we assigned the $H N F-6$ gene to chromosome $15,2.2 \mathrm{cR}_{3000}$ downstream from marker WI2833 and $13.8 \mathrm{cR}_{3000}$ upstream from marker AFMB342WD9 (primer set 1; lod > 3.0). Mapping with a second primer set located the HNF-6 gene at $5.55 \mathrm{cR}_{3000}$ from WI2833 and $10.5 \mathrm{cR}_{3000}$ from AFMB342WD9 (lod > 3.0). We then cloned and sequenced the coding region and the exon/intron junctions of the human $H N F-6$ gene. A comparison of the full-length rat $H N F-6$ cDNA [6] and gene [17] with the partial cDNA for human $H N F-6$ [8] showed that this partial cDNA included the coding sequence from amino acid 174 to the end (amino acid 465). It also predicted that the coding portion of the human gene was made of only two exons, with a splice site between residues 368 and 369 . The first exon codes for the $\mathrm{NH}_{2}$-terminal part of HNF-6, which includes the cut domain (residues 292 to 357). The other exon codes for the rest of the protein, which includes the homeodomain (residues 385 to 444).

Single strand conformational polymorphism-heteroduplex analysis of the HNF-6 gene in $6 \mathrm{MODY}$ patients without mutations in the MODY1, MODY3 and MODY4 genes and in 54 patients with Type II diabetes of late onset showed one missense variant at codon 75 (Pro $\rightarrow$ Ala) and two silent variants at codon 94, Pro(CCC $\rightarrow$ CCG) and at codon 287, Gly(GGG $\rightarrow$ GGT). The Pro94Pro variant was a common polymorphism and was found in 18 of the 60 patients examined. The Gly287Gly variant was only identified in one of the patients examined. None of the silent variants were examined further.
The allelic frequency of the Pro75Ala variant was $3.2 \%$ (95\% confidence interval: $1.9-4.5$ ) in 330 Type II diabetic patients compared with $4.2 \%(2.4-6.0)$ in 238 glucose tolerant control subjects $(p=\mathrm{NS})$. None of the allelic frequencies were statistically significantly different from Hardy-Weinberg equilibrium. No homozygous carrier of this variant was identified. At the phenotype level there were no statistically significant differences in age of onset, BMI, fasting concentrations of plasma glucose, serum $\mathrm{C}$ peptide and serum insulin between carriers and non-carriers of the Pro75 Ala variant within the group of 330 Type II diabetic patients (data not shown). Neither were there any statistically significant differences between genotype groups in age, BMI, sex and 30-min concentrations of plasma glucose, serum insulin and serum $\mathrm{C}$ peptide during an OGTT in the group of 238 glucose tolerant carriers and non-carriers of the Pro75Ala polymorphism (Table 1 ).

In the group of 367 young healthy Caucasians the allelic frequency of the Pro75 Ala variant was $2.0 \%$ (1.0-3.0). No statistically significant differences between carriers and non-carriers of the variant were identified in fasting concentrations of plasma glucose, serum insulin and $\mathrm{C}$ peptide or immediate insulin and $\mathrm{C}$ peptide responses after intravenous glucose injection (Table 1). In contrast, the variant was associated with increased body mass index $(p=0.003)$ in this population sample and when adjusting for differences in age and sex in a regression analysis, the association remained $(p=0.04)$. Likewise, young heterozygous carriers of the variant had decreased insulin sensitivity compared with wild-type carriers $(p=0.04)$, a difference that remained after adjustment for differences between the two groups in age, sex and body mass index $(p=0.04)$.

Among the 226 glucose tolerant offspring of Type II diabetic patients the codon 75 polymorphism had an allelic frequency of $4.6 \%(2.7-6.5)$. The variant had no impact on the measured prediabetic phenotypes (Table 1).

\section{Discussion}

To carry out an analysis for mutations of the HNF- 6 gene in Type II diabetic patients, we have cloned the coding region and characterized the exon/intron junctions of the human HNF-6 gene. We found that the HNF-6 protein is coded by two exons, with a splice site between residues 368 and 369. The first exon codes for the $\mathrm{NH}_{2}$-terminal part of HNF-6, which includes the cut domain (residues 292 to 357). The other exon codes for the rest of the protein, which includes the homeodomain (residues 385 to 444). The chromosomal localisation of the human HNF-6 gene has previously been established by fluorescence in situ hybridization to 15q21.1-21.2 [11]. Our radiation 
hybrid analysis confirmed and extended these data [11].

The sequences obtained allowed us to screen for variability of the HNF-6 gene in 6 MODY patients without mutations in the MODY1, MODY3 and MODY4 genes and in 54 patients with Type II diabetes of late onset. This showed one missense variant at codon 75 (Pro $\rightarrow$ Ala) and two silent variants at codon 94 and at codon 287. The missense polymorphism results in replacement by an aliphatic amino acid of a cyclic amino acid which is conserved in the human, rat and mouse HNF-6. Residue 75 is located in the sequence of the protein upstream from the two DNA binding domains, in a region of HNF-6 of unknown function. The allelic frequency of the Pro75Ala variant were not statistically significantly different between Type II diabetic patients and glucose tolerant control subjects. The variant was associated neither with differences in age at clinical diabetes onset, BMI, fasting concentrations of plasma glucose, serum $\mathrm{C}$ peptide and serum insulin within the group of 330 Type II diabetic patients nor with differences in measurements of beta-cell function in 238 middle-aged, glucose tolerant subjects, in 226 glucose tolerant offspring of Type II diabetic patients or in 367 young healthy subjects. The variant was, however, associated with increased body mass index in the group of young healthy subjects $(p=0.003)$; the association remained after adjustment for sex and age in a regression analysis $(p=0.04)$. Also, young heterozygous carriers of the variant had decreased insulin sensitivity compared with wild-type carriers $(p=0.04)$, a difference that remained statistically significant after adjustment for differences between the two groups in age, sex and body mass index $(p=0.04)$. As our primary hypothesis in this study was the existence of a relation between variability in the HNF-6 gene and insulin secretion, the difference in BMI and insulin sensitivity between carriers and non-carriers of the Pro75Ala variant among the 367 young healthy subjects disappeared after correction for multiple testing. Also, the differences in BMI and insulin sensitivity index could not be found in the group of glucose tolerant offspring of Type II diabetic probands. For this reason we consider the positive associations in the group of young subjects as incidental findings.

Assuming a $30 \%$ effect of the variant on the key variables we have calculated the power of both groups studied (young healthy subjects and first degree relatives of Type II diabetic patients) to detect differences in the variables to be between $40 \%$ and $80 \%$ for immediate serum insulin and C-peptide responses. Therefore, our study materials did not have sufficient power to exclude a possible minor biological effect of the Pro75Ala variant in heterozygous form.

In conclusion, we have examined the coding region and intron-exon junctions of $H N F-6$ for genetic variability in Danish Caucasian Type II diabetic patients and have identified two silent and one missense (Pro75Ala) polymorphisms. The Pro75Ala polymorphism was not associated with Type II diabetes or with impaired insulin secretion in glucose tolerant subjects.

Acknowledgements. The study was supported by grants from the University of Copenhagen, the Danish Medical Research Council, the Velux Foundation, the Danish Diabetes Association, EEC grant (BMH4-CT-950 662) and by Belgian grants from the Interuniversity Poles of Attraction, the D. G. Higher Education and Scientific Research, the National Fund for Scientific Research (Belgium) and the Fund for Scientific Medical Research (Belgium). The authors thank M. Parmentier for the genomic library, C. Szpirer for oligonucleotides, G. Vassart and M. Vikkula for advice, S. Urioste, A. Forman, L. Aabo, B. Mottlau, S. Kjellberg, J. Brønnum and Q. Truong for dedicated and careful technical assistance and G. Lademann for secretarial support. F.P. Lemaigre. is Senior Research Associate of the National Fund for Scientific Research (Belgium).

\section{References}

1. Cereghini S (1996) Liver-enriched transcription factors and hepatocyte differentiation. FASEB J 10: 267-282

2. Yamagata K, Furuta H, Oda N et al. (1996) Mutations in the hepatocyte nuclear factor- $4 \alpha$ gene in maturity-onset diabetes of the young (MODY1). Nature 384: 458-460

3. Yamagata K, Oda N, Kaisaki PJ et al. (1996) Mutations in the hepatocyte nuclear factor-1 $\alpha$ gene in maturity-onset diabetes of the young (MODY3). Nature 384: 455-458

4. Horikawa Y, Iwasaki N, Hara M et al. (1997) Mutations in the hepatocyte nuclear factor- $1 \beta$ gene (TCF2) associated with MODY. Nat Genet 17: 384-385

5. Lannoy VJ, Bürglin TR, Rousseau GG, Lemaigre FP (1998) Isoforms of hepatocyte nuclear factor-6 differ in DNA-binding properties, contain a bifunctional homeodomain, and define the new ONECUT class of homeodomain proteins. J Biol Chem 273: 13552-13562

6. Lemaigre FP, Durviaux SM, Truong O, Lannoy VJ, Hsuan JJ, Rousseau GG (1996) Hepatocyte nuclear factor 6, a transcription factor that contains a novel type of homeodomain and a single cut domain. Proc Natl Acad Sci USA 93: 9460-9464

7. Landry C, Clotman F, Hioki T et al. (1997) HNF-6 is expressed in endoderm derivatives and nervous system of the mouse embryo and participates to the cross-regulatory network of liver-enriched transcription factors. Dev Biol 192: 247-257

8. Rausa F, Samadani U, Ye H et al. (1997) The cut-homeodomain transcription activator HNF-6 is coexpressed with its target gene HNF-3 $\beta$ in the developing murine liver and pancreas. Dev Biol 192: 228-246

9. Wu KL, Gannon M, Peshavaria M et al. (1997) Hepatocyte nuclear factor 3 beta is involved in pancreatic beta-cell specific transcription of the pdx-1 gene. Mol Cell Biol 17: 6002-6013

10. Stoffers DA, Ferrer J, Clarke WL, Habener JF (1997) Early-onset type-II diabetes mellitus (MODY4) linked to IPF1. Nat Genet 17: 138-139

11. Vaisse C, Kim J, Espinosa R, Le Beau MM, Stoffel M (1997) Pancreatic islet expression studies and polymorphic 
DNA markers in the genes encoding hepatocyte nuclear factor-3 $\alpha,-3 \beta, 3 \gamma$ and -6 . Diabetes 46: 1364-1367

12. Andersen L, Dinesen B, Jørgensen PN, Poulsen F, Røder ME (1993) Enzyme immunoassay for intact human insulin in serum and plasma. Clin Chem 39: 578-582

13. Heding LG (1975) Radioimmunological determination of human C-peptide in serum. Diabetologia 11: 541-548

14. Faber OK, Marcussen J, Naithani VK, Binder C (1976) Production of antisera to synthetic benzyloxycarbonyl-Cpeptide. Hoppe-Seylor's Z Physiol Chem 357: 751-757

15. Faber OK, Binder C, Marcussen J et al. (1978) Characterization of seven C-peptide antisera. Diabetes 27 [Suppl. 1]: 170-177

16. Clausen JO, Borch-Johnsen K, Ibsen H et al. (1996) Insulin sensivity index, acute insulin response and glucose effectiveness in a population-based sample of 380 young healthy
Caucasians. Analysis of the impact of gender, body fat, physical fitness and lifestyle factors. J Clin Invest 98: 1195-1209

17. Rastegar M, Szpirer C, Rousseau GG, Lemaigre FP (1998) Hepatocyte nuclear factor-6: organization and chromosomal assignment of the rat gene and characterization of its promoter. Biochem J 334: 565-569

18. Hansen T, Andersen CB, Echwald SM et al. (1997) Identification of a common amino acid polymorphism in the p85 a regulatory subunit of phosphatidylinositol 3-kinase. Effects on glucose disappearance constant, glucose effectiveness and the insulin sensitivity index. Diabetes 46: 494-501

19. Hooper JL, Mathews JD (1982) Extension to multivariate normal models for pedigree analysis. Ann Hum Genet 46: $373-383$ 